\title{
Generalized k- Derivations on Lie Ideals of Prime $\Gamma$-Rings
}

\author{
A. C. Paul ${ }^{1}$ and Ayesha Nazneen ${ }^{2}$ \\ Department of Mathematics, Rajshahi University Rajshahi - 6205, Bangladesh
}

\begin{abstract}
Let $M$ be a 2-torsion free prime $\Gamma$-ring and $U$ a Lie ideal of $M$. Let $F: M \rightarrow M$ be a mapping defined by $F(u \alpha v)=F(u) \alpha v+u k(\alpha) v+u \alpha d(v)$, for all $u, v \in U$ and $\alpha \in \Gamma$. Then $F$ is a generalized $k$ derivation on $U$ of $M$ if there exists a $k$ - derivation $d$ on $U$ of $M$. Also $F$ is a Jordan generalized $k$ - derivation on $U$ of $M$ if there exists a $k$ - derivation $d$ on $U$ of $M$ such that $F(u \alpha u)=F(u) \alpha u+u k(\alpha) u+u \alpha d(u)$, for all $u \in$ $U$ and $\alpha \in \Gamma$. In this article, we prove that every Jordan generalized $k$ - derivation on a Lie ideal $U$ of a 2 torsion free prime $\Gamma$-ring $M$ is a generalized $k$-derivation on $U$ of $M$.
\end{abstract}

Keywords: Lie ideal, $k$ - derivation, generalized $k$ - derivation; Jordan generalized $k$ - derivation, Prime $\Gamma$ ring.

\section{Introduction}

The $\Gamma$ - ring is a generalized form of a ring. Nobusawa [1] and Barnes [2] developed the concept of a $\Gamma$ ring. The definition of a $\Gamma$ - ring is as follows :

Let $\mathrm{M}$ and $\Gamma$ be two additive abelian groups. If there is a mapping $\mathrm{M} \times \Gamma \times \mathrm{M} \rightarrow \mathrm{M}$ defined by $(\mathrm{x}, \alpha, \mathrm{y}) \rightarrow \mathrm{x} \alpha \mathrm{y}$ such that ;

(a) $(x+y) \alpha z=x \alpha z+y \alpha z, x(\alpha+\beta) y=x \alpha y+x \beta y, x \alpha(y+z)=x \alpha y+x \alpha z$ and

(b) (xay) $\beta z=x \alpha(y \beta z)$ are satisfied for all $x, y, z \in M$ and $\alpha \in \Gamma$, then $M$ is called a $\Gamma$ - ring in the sense of Bernes [2]. Throughout the paper, we use $\mathrm{M}$ as a $\Gamma$ - ring.

In addition to the definition given above, if there is a mapping $\Gamma \times \mathrm{M} \times \Gamma \rightarrow \Gamma$ satisfying

$\left(\mathrm{a}^{*}\right)(\alpha+\beta) \mathrm{x} \gamma=\alpha \mathrm{x} \gamma+\beta \mathrm{x} \gamma, \alpha(\mathrm{x}+\mathrm{y}) \beta=\alpha \mathrm{x} \beta+\alpha \mathrm{y} \beta, \alpha \mathrm{x}(\beta+\gamma)=\alpha \mathrm{x} \beta+\alpha \mathrm{x} \gamma$

$\left(b^{*}\right)(x \alpha y) \beta z=x(\alpha y \beta) z=x \alpha(y \beta z)$;

(c*) $\mathrm{x} \alpha \mathrm{y}=0$ implies $\alpha=0$, for all $\mathrm{x}, \mathrm{y}, \mathrm{z} \in \mathrm{M}$ and $\alpha, \beta, \gamma \in \Gamma$; then $\mathrm{M}$ is called a $\Gamma$ - ring in the sense of Nobusawa [1] as simply a $\Gamma_{N}$ - ring. It is clear that $\mathrm{M}$ is a $\Gamma_{N}$ - ring implies that $\Gamma$ is an $\mathrm{M}$-ring. $\mathrm{M}$ is called a prime $\Gamma$ - ring, if for all $\mathrm{x}, \mathrm{y} \in \mathrm{M}, \mathrm{x} \Gamma \mathrm{M} \Gamma \mathrm{y}=0$ implies $\mathrm{x}=0$ or $\mathrm{y}=0$. And $\mathrm{M}$ is called a semiprime $\Gamma$ - ring if for all $\mathrm{x} \in \mathrm{M}, \mathrm{x} \Gamma \mathrm{M} \Gamma \mathrm{x}=0$ implies $\mathrm{x}=0$. It is clear that every prime $\Gamma$ - ring is also semi prime but the converse is not true in general. Also $M$ is called a 2 - torsion free if $2 x=0$ implies $x=0$ for every $x \in M$.

The concept of derivations and Jordan derivations were introduced by M. Sapanci and A. Nakajima [3]. H. Kandamar [4] has developed the k-derivation of a $\Gamma$ - ring. The notion of Jordan k- derivation of a $\Gamma$ - ring was first introduced by S. Chakraborty and A. C. Paul [5] and proved that every Jordan k- derivation on a 2- torsion free prime $\Gamma_{N}$ - ring $\mathrm{M}$ is a k- derivation on $\mathrm{M}$. The generalized derivations of a $\Gamma$-ring was introduced by $\mathrm{Y}$. Ceven and M. A. Ozturk [6] and proved that every Jordan generalized derivation of a $\Gamma$ - ring $M$ is a generalized derivation of M. M. M. Rahman and A. C. Paul [7] extended the results of [6] on Lie ideals of prime $\Gamma$ - rings. In [8], S. Uddin and Paul worked on simple $\Gamma$-rings with involutions and extended various results of Herstein [9] in $\Gamma$ - rings. S. Chakraborty and A. C. Paul $[10,11,12,13,14,15]$ worked on Jordan generalized k-derivations on prime $\Gamma_{N}$ - rings, completely prime and completely semiprime $\Gamma_{N}$ - rings and developed the various significant results on these fields. The definition of a k- derivation and a Jordan k- derivation are as follows:

Let $\mathrm{M}$ be a $\Gamma$ - ring. Let $\mathrm{d}: \mathrm{M} \rightarrow \mathrm{M}$ and $\mathrm{k}: \Gamma \rightarrow \Gamma$ be an additive mappings. If $\mathrm{d}(\mathrm{x} \alpha \mathrm{y})=\mathrm{d}(\mathrm{x}) \alpha \mathrm{y}+\mathrm{xk}(\alpha) \mathrm{y}+\mathrm{x} \alpha \mathrm{d}(\mathrm{y})$ is satisfied for all $\mathrm{x}, \mathrm{y} \in \mathrm{M}$ and $\alpha \in \Gamma$, then $\mathrm{d}$ is said to be a k- derivation of $\mathrm{M}$.

Example : Let $\mathrm{R}$ be an associative Ring. Define $M=M_{1,2}(R)$ and $\Gamma=\mathrm{M}_{2,1}(R)$. Then $\mathrm{M}$ is a $\Gamma$ - ring.

Define $\mathrm{d}: \mathrm{M} \rightarrow \mathrm{M}$ by $\mathrm{d}((\mathrm{a}, \mathrm{b}))=(0, \mathrm{~b})$ and $\mathrm{k}: \Gamma \rightarrow \Gamma$ by $k\left(\left(\begin{array}{l}\alpha \\ \beta\end{array}\right)\right)=\left(\begin{array}{l}0 \\ -\beta\end{array}\right)$.

Then $\mathrm{d}$ is a $\mathrm{k}$ - derivation of $\mathrm{M}$ for,

$(0, b)\left(\begin{array}{l}\alpha \\ \beta\end{array}\right)(x, y)+(a, b)\left(\begin{array}{l}0 \\ -\beta\end{array}\right)(x, y)+(a, b)\left(\begin{array}{l}\alpha \\ \beta\end{array}\right)(0, y)$ 


$$
\begin{aligned}
& =(b \beta x, b \beta y)+(-b \beta x,-b \beta y)+(0, a \alpha y+b \beta y) \\
& =(b \beta x-b \beta x+0, b \beta y-b \beta y+a \alpha y+b \beta y) \\
& =(0, a \alpha y+b \beta y) \\
& \text { Also }(a, b)\left(\begin{array}{l}
\alpha \\
\beta
\end{array}\right)(x, y)=(a \alpha x+b \beta x, a \alpha y+b \beta y) \\
& \Rightarrow d\left((a, b)\left(\begin{array}{l}
\alpha \\
\beta
\end{array}\right)(x, y)\right)=d((a \alpha x+b \beta x, a \alpha y+b \beta y)) \\
& =(0, a \alpha y+b \beta y) \\
& =d((a, b))\left(\begin{array}{l}
\alpha \\
\beta
\end{array}\right)(x, y)+(a, b) k\left(\left(\begin{array}{l}
\alpha \\
\beta
\end{array}\right)\right)(x, y)+(a, b)\left(\begin{array}{l}
\alpha \\
\beta
\end{array}\right) d((0, y))
\end{aligned}
$$

And if $\mathrm{d}(\mathrm{x} \alpha \mathrm{x})=\mathrm{d}(\mathrm{x}) \alpha \mathrm{x}+\mathrm{xk}(\alpha) \mathrm{x}+\mathrm{x} \alpha \mathrm{d}(\mathrm{x})$ holds for every $\mathrm{x} \in \mathrm{M}$ and $\alpha \in \Gamma$, then $\mathrm{d}$ is said to be Jordan $\mathrm{k}$ derivation of $\mathrm{M}$. Note that every k-derivation is a Jordan $\mathrm{k}$ - derivation but the converse is not true always. Here the notation $[x, y]_{\alpha}$ is used for the commutator $\mathrm{x}$ and $\mathrm{y}$ with respect to $\alpha$, which is defined by $[x, y]_{\alpha}=x \alpha y-y \alpha x$. If $\mathrm{A}$ is a subset of $\mathrm{M}$, the centre of $\mathrm{A}$ with respect to $\mathrm{M}$ is $\mathrm{Z}(\mathrm{A})$ and is defined by $Z(A)=\left\{x \in M:[x, a]_{\alpha}=0\right.$ for all $a \in A$ and $\left.\alpha \in \Gamma\right\}$. The centre of a $\Gamma$ - ring $\mathrm{M}$ is denoted by $\mathrm{Z}(\mathrm{M})$ and is defined by $Z(M)=\left\{x \in M:[x, y]_{\alpha}=0\right.$ for all $\mathrm{y} \in \mathrm{M}$ and $\left.\alpha \in \Gamma\right\}$. A $\Gamma$ - ring $\mathrm{M}$ is commutative if and only if $\mathrm{M}=\mathrm{Z}(\mathrm{M})$.

Throughout this paper, we shall use the condition (*) a $\alpha \mathrm{b} \beta \mathrm{c}=\mathrm{a} \beta \mathrm{b} \alpha \mathrm{c}$ for all $\mathrm{a}, \mathrm{b}, \mathrm{c} \in \mathrm{M}$ and $\alpha, \beta \in \Gamma$. By the condition, the commutator identities

$[a \alpha b, x]_{\beta}=[a, x]_{\beta} \alpha b+a[\alpha, \beta]_{x} b+a \alpha[b, x]_{\beta}$ and $[\mathrm{x}, \mathrm{a} \alpha \mathrm{b}]_{\beta}=a \alpha[x, b]_{\beta}+a[\alpha, \beta]_{\beta} b+[x, a]_{\beta} \alpha \beta$ given in [4] reduces to $[a \alpha b, x]_{\beta}=a \alpha[b, x]_{\beta}+[a, x]_{\beta} \alpha b$ and $[x, a \alpha b]_{\beta}=a \alpha[x, b]_{\beta}+[x, a]_{\beta} \alpha b$.

In this paper, we prove that every Jordan generalized k- derivation on a Lie ideal $\mathrm{U}$ of $\mathrm{M}$ is a generalized kderivation on $\mathrm{U}$ of $\mathrm{M}$.

\section{Generalized and Jordan Generalized k- Derivation}

Definition 2.1. Let $M$ be a $\Gamma$-ring and let $k: \Gamma \rightarrow \Gamma$ be an additive mapping. An additive mapping $F: M \rightarrow M$ is called a generalized k- derivation if there exists a k- derivation $\mathrm{d}: \mathrm{M} \rightarrow \mathrm{M}$ such that $\mathrm{F}(\mathrm{u} \alpha \mathrm{v})=\mathrm{F}(\mathrm{u}) \alpha \mathrm{v}+\mathrm{uk}(\alpha) \mathrm{v}+$ $\mathrm{u} \alpha \mathrm{d}(\mathrm{v})$ for all $\mathrm{u}, \mathrm{v} \in \mathrm{M}$ and $\alpha \in \Gamma$. And if $\mathrm{F}(\mathrm{u} \alpha \mathrm{u})=\mathrm{F}(\mathrm{u}) \alpha \mathrm{u}+\mathrm{uk}(\alpha) \mathrm{u}+\mathrm{u} \alpha \mathrm{d}(\mathrm{u})$ holds for all $\mathrm{u} \in \mathrm{M}$ and $\alpha \in \Gamma$, then $\mathrm{F}$ is said to be a Jordan generalized $\mathrm{k}$ - derivation.

Example : Let $\mathrm{M}$ be a $\Gamma$ - ring and let $\mathrm{F}$ be a generalized $\mathrm{k}$ - derivation of $\mathrm{M}$.Then by definition, there exists a kderivation $d: M \rightarrow M$ such that $d(x \alpha y)=d(x) \alpha y+x k(\alpha) y+x \alpha d(y)$ and $F(x \alpha y)=F(x) \alpha y+x k(\alpha) y+x \alpha d(y)$, for all $\mathrm{x}, \mathrm{y} \in \mathrm{M}$ and $\alpha \in \Gamma$.

Let $M_{1}=M \times M$ and $\Gamma_{1}=\Gamma \times \Gamma$. Define the operations of addition and multiplication of $M_{1}$ and $\Gamma_{1}$ by $(\mathrm{x}, \mathrm{y})+(\mathrm{z}, \mathrm{w})=(\mathrm{x}+\mathrm{z}, \mathrm{y}+\mathrm{w})$ and $(\mathrm{x}, \mathrm{y})(\alpha, \beta)(\mathrm{z}, \mathrm{w})=(\mathrm{x} \alpha \mathrm{z}, \mathrm{y} \beta \mathrm{w})$ for every $\mathrm{x}, \mathrm{y}, \mathrm{z}, \mathrm{w} \in \mathrm{M}$ and $\alpha, \beta \in \Gamma$. Then $M_{1}$ is obviously a $\Gamma_{1}$ - ring under these operations.

Let $\quad F_{1}: M_{1} \rightarrow M_{1}, d_{1}: M_{1} \rightarrow M_{1}$ and $k_{1}: \Gamma_{1} \rightarrow \Gamma_{1} \quad$ be the additive mappings defined by $F_{1}((x, y))=(F(x), F(y)), \quad d_{1}((x, y))=(d(x), d(y)) \quad$ and $\quad k_{1}((\alpha, \beta))=(k(\alpha), k(\beta))$

for all $\mathrm{x}, \mathrm{y} \in \mathrm{M}$ and $\alpha, \beta \in \Gamma$. Then clearly $d_{1}$ is a $k_{1}$-derivation of $M_{1}$.

Put $(x, y)=a \in M_{1},(\alpha, \beta)=\gamma \in \Gamma_{1}$, for any $\mathrm{x}, \mathrm{y} \in \mathrm{M}$ and $\alpha, \beta \in \Gamma$; then we have, 


$$
\begin{aligned}
& F_{1}(a \gamma a)=F_{1}((x, y)(\alpha, \beta)(x, y)) \\
& =F_{1}(x \alpha x, y \beta y) \\
& =(F(x \alpha x), F(y \beta y)) \\
& =(F(x) \alpha x+x k(\alpha) x+x \alpha d(x), F(y) \beta y+y k(\beta) y+y \beta d(y)) \\
& =(F(x) \alpha x, F(y) \beta y)+(x k(\alpha) x, y k(\beta) y)+(x \alpha d(x), y \beta d(y)) \\
& =(F(x), F(y))(\alpha, \beta)(x, y)+(x, y)(k(\alpha), k(\beta))(x, y)+(x, y)(\alpha, \beta)(d(x), d(y)) \\
& =F_{1}(x, y)(\alpha, \beta)(x, y)+(x, y) k_{1}(\alpha, \beta)(x, y)+(x, y)(\alpha, \beta) d_{1}(x, y) \\
& =F_{1}(a) \gamma a+a k_{1}(\gamma) a+a \gamma d_{1}(a),
\end{aligned}
$$

which follows that $F_{1}$ is a Jordan generalized $k_{1}$-derivation of $M_{1}$ associated with the $k_{1}$-derivation $d_{1}$ of $M_{1}$.

Definition 2.2. Let $\mathrm{M}$ be a $\Gamma$ - ring. An additive subgroup $\mathrm{U}$ of $\mathrm{M}$ is called a Lie ideal of $\mathrm{M}$ if $[u, m]_{\alpha} \in U$ for every $\mathrm{u} \in \mathrm{U}, \mathrm{m} \in \mathrm{M}$ and $\alpha \in \Gamma$. Note that every ideal of a $\Gamma$ - ring $\mathrm{M}$ is a Lie ideal of $\mathrm{M}$ but the converse is not true in general.

Example: Let $\mathrm{R}$ be a ring and $\mathrm{U}$ be a Lie ideal of $\mathrm{R}$. Let $M=M_{1,2}(\mathrm{R})$ and $\Gamma=\left\{\left(\begin{array}{l}n \cdot 1 \\ 0\end{array}\right): n \in Z\right\}$. Then $\mathrm{M}$ is a $\Gamma$ - ring . Define $\mathrm{N}=\{(\mathrm{x}, \mathrm{x}): \mathrm{x} \in \mathrm{R}\} \subseteq \mathrm{M}$. Then $\mathrm{N}$ is a $\Gamma$ - ring. Let $U_{1}=\{(u, u): u \in U\}$.

$$
\begin{aligned}
& \text { Now }(u, u)\left(\begin{array}{l}
n \\
0
\end{array}\right)(a, a)-(a, a)\left(\begin{array}{l}
n \\
0
\end{array}\right)(u, u) \\
& =(\text { una }, \text { una })-(\text { anu }, \text { anu }) \\
& =(\text { una }- \text { anu }, \text { una }- \text { anu }) \in U_{1} .
\end{aligned}
$$

Then $U$ is a Lie ideal of $\mathrm{N}$. It is clear that $U_{1}$ is not an ideal of $\mathrm{N}$.

Definition 2.3. Let $\mathrm{M}$ be a $\Gamma$ - ring and let $\mathrm{U}$ be a Lie ideal of $\mathrm{M}$. Let $\mathrm{k}: \Gamma \rightarrow \Gamma$ be an additive mapping. An additive mapping $\mathrm{F}: \mathrm{M} \rightarrow \mathrm{M}$ is called a generalized $\mathrm{k}$ - derivation on $\mathrm{U}$ of $\mathrm{M}$ if there exists a k- derivation $\mathrm{d}$ on $\mathrm{U}$ of $\mathrm{M}$ such that $\mathrm{F}(\mathrm{u} \alpha \mathrm{v})=\mathrm{F}(\mathrm{u}) \alpha \mathrm{v}+\mathrm{uk}(\alpha) \mathrm{v}+\mathrm{u} \alpha \mathrm{d}(\mathrm{v})$ for all $\mathrm{u}, \mathrm{v} \in \mathrm{U}$ and $\alpha \in \Gamma$.

Example: Let $\mathrm{M}$ be a $\Gamma$ - ring and let $\mathrm{U}$ be a Lie ideal of $\mathrm{M}$. Let $\mathrm{f}: \mathrm{M} \rightarrow \mathrm{M}$ be a generalized $\mathrm{k}$ - derivation on $\mathrm{U}$ of $\mathrm{M}$, then there exists a derivation $\mathrm{d}$ on $\mathrm{U}$ of $\mathrm{M}$ such that $\mathrm{f}(\mathrm{u} \alpha \mathrm{v})=\mathrm{f}(\mathrm{u}) \alpha \mathrm{v}+\mathrm{uk}(\alpha) \mathrm{v}+\mathrm{u} \alpha \mathrm{d}(\mathrm{v})$ for all $\mathrm{u}, \mathrm{v} \in \mathrm{U}$ and $\alpha \in \Gamma$. Let $M_{1}=\{(x, x): x \in M\}$ and $\Gamma_{1}\{(\alpha, \alpha): \alpha \in \Gamma\}$. Define addition and multiplication of $\mathrm{M}$ are as follows:

$(\mathrm{x}, \mathrm{x})+(\mathrm{y}, \mathrm{y})=(\mathrm{x}+\mathrm{y}, \mathrm{x}+\mathrm{y}),(\mathrm{x}, \mathrm{x})(\alpha, \alpha)(\mathrm{y}, \mathrm{y})=(\mathrm{x} \alpha \mathrm{y}, \mathrm{x} \alpha \mathrm{y})$ for all $(x, x) \in M_{1}$ and $(\alpha, \alpha) \in \Gamma_{1}$. Under these operations $M_{1}$ is a $\Gamma_{1}$ - ring.

Let $U_{1}=\{(u, u): u \in U\}$. Then clearly $U_{1}$ is a Lie ideal of $M_{1}$. Define $F: M_{1} \rightarrow M_{1}, D: M_{1} \rightarrow M_{1}$ and $k_{1}: \Gamma_{1} \rightarrow \Gamma_{1}$ by $F((x, x))=(f(x), f(x)), D((x, x))=(d(x), d(x))$ and $\mathrm{k}_{1}((\alpha, \alpha))=(k(\alpha), k(\alpha))$ for all $x \in U$ and $\alpha \in \Gamma$.

Then $F((x, x)(\alpha, \alpha)(y, y))=F(x \alpha y, x \alpha y))$

$=(f(x \alpha y), f(x \alpha y))$

$=(f(x) \alpha y+x k(\alpha) y+x \alpha d(y), f(x) \alpha y+x k(\alpha) y+x \alpha d(y))$

$=(f(x) \alpha y, f(x) \alpha y))+(x k(\alpha) y, x k(\alpha) y)+(x \alpha d(y), x \alpha d(y))$ 


$$
\begin{aligned}
& =(f(x), f(x))(\alpha, \alpha)(y, y)+(x, x)(k(\alpha), k(\alpha))(y, y)+(x, x)(\alpha, \alpha)(d(y), d(y)) \\
& =F((x, x))(\alpha, \alpha)(y, y)+(x, x) k_{1}((\alpha, \alpha))(y, y)+(x, x)(\alpha, \alpha) D((y, y))
\end{aligned}
$$

Therefore $\mathrm{F}$ is a generalized k- derivation on $U_{1}$ of $M_{1}$.

Also $\mathrm{F}: \mathrm{M} \rightarrow \mathrm{M}$ is called a Jordan generalized k- derivation on $\mathrm{U}$ of $\mathrm{M}$ if there exist a $\mathrm{k}$ - derivation $\mathrm{d}$ on $\mathrm{U}$ of $\mathrm{M}$ such that $\mathrm{F}(\mathrm{u} \alpha \mathrm{u})=\mathrm{F}(\mathrm{u}) \alpha \mathrm{u}+\mathrm{uk}(\alpha) \mathrm{u}+\mathrm{u} \alpha \mathrm{d}(\mathrm{u})$, for every $\mathrm{u} \in \mathrm{U}$ and $\alpha \in \Gamma$.

Example: Let $\mathrm{M}$ be a $\Gamma$ - ring and let $\mathrm{U}$ be a Lie ideal of $\mathrm{M}$. Let $\mathrm{f}: \mathrm{M} \rightarrow \mathrm{M}$ be a generalized $\mathrm{k}$ - derivation on $\mathrm{U}$ of $M$, then there exists a derivation $d$ on $U$ of $M$ such that $f(u \alpha v)=f(u) \alpha v+u k(\alpha) v+u \alpha d(v)$ for all $u, v \in U$ and $\alpha \in \Gamma$.

Let $M_{1}=\{(x, x): x \in M\}$ and $\Gamma_{1}:\{(\alpha, \alpha): \alpha \in \Gamma\}$. Define addition and multiplication of $\mathrm{M}$ are as follows:

$(\mathrm{x}, \mathrm{x})+(\mathrm{y}, \mathrm{y})=(\mathrm{x}+\mathrm{y}, \mathrm{x}+\mathrm{y}),(\mathrm{x}, \mathrm{x})(\alpha, \alpha)(\mathrm{y}, \mathrm{y})=(\mathrm{x} \alpha \mathrm{y}, \mathrm{x} \alpha \mathrm{y})$ for all $(x, x) \in M_{1}$ and $(\alpha, \alpha) \in \Gamma_{1}$. Under these operations $M_{1}$ is a $\Gamma_{1}$ - ring. Let $U_{1}=\{(u, u): u \in U\}$. Then clearly $U_{1}$ is a Lie ideal of $M_{1}$. Define

$F: M_{1} \rightarrow M_{1}, D: M_{1} \rightarrow M_{1}$ and $k_{1}: \Gamma_{1} \rightarrow \Gamma_{1}$ by $F((x, x))=(f(x), f(x)), D((x, x)=(d(x), d(x))$ and $k_{1}((\alpha, \alpha))=(k(\alpha), k(\alpha))$ for all $x \in U$ and $\alpha \in \Gamma$.

Then $F((x, x)(\alpha, \alpha)(x, x))=F((x \alpha x, x \alpha x))$

$=(f(x \alpha x), f(x \alpha x))$

$=(f(x) \alpha x+x k(\alpha) x+x \alpha d(x), f(x) \alpha x+x k(\alpha) x+x \alpha d(x))$

$=(f(x) \alpha x, f(x) \alpha x)+(x k(\alpha) x, x k(\alpha) x)+(x \alpha d(x), x \alpha d(x))$

$=(f(x), f(x))(\alpha, \alpha)(x, x)+(x, x)(k(\alpha), k(\alpha))(x, x)+(x, x)(\alpha, \alpha)(d(x), d(x))$

$=F((x, x))(\alpha, \alpha)(x, x)+(x, x) k_{1}((\alpha, \alpha))(x, x)+(x, x)(\alpha, \alpha) D((x, x))$.

Therefore $\mathrm{F}$ is a Jordan generalized k- derivation on $U_{1}$ of $M_{1}$.

Lemma 2.4 Let $\mathrm{M}$ be a 2- torsion free $\Gamma$-ring satisfying $(*)$ and $\mathrm{U}$ a Lie ideal of $\mathrm{M}$ such that $\mathrm{u \alpha u} \in \mathrm{U}$ for all $\mathrm{u} \in \mathrm{U}$ and . Let $\mathrm{F}: \mathrm{M} \rightarrow \mathrm{M}$ be a Jordan generalized k- derivation on $\mathrm{U}$, then

(i) $\quad \mathrm{F}(\mathrm{u} \alpha \mathrm{v}+\mathrm{v} \alpha \mathrm{u})=\mathrm{F}(\mathrm{u}) \alpha \mathrm{v}+\mathrm{uk}(\alpha) \mathrm{v}+\mathrm{u} \alpha \mathrm{d}(\mathrm{v})+\mathrm{F}(\mathrm{v}) \alpha \mathrm{u}+\mathrm{vk}(\alpha) \mathrm{u}+\mathrm{v} \alpha \mathrm{d}(\mathrm{u})$.

(ii) $\quad \mathrm{F}(\mathrm{u} \alpha \mathrm{v} \beta \mathrm{u})=\mathrm{F}(\mathrm{u}) \alpha v \beta \mathrm{u}+\mathrm{uk}(\alpha) \mathrm{v} \beta \mathrm{u}+\mathrm{u} \alpha \mathrm{d}(\mathrm{v}) \beta \mathrm{u}+\mathrm{u} \alpha \mathrm{vk}(\beta) \mathrm{u}+\mathrm{u} \alpha \mathrm{v} \beta \mathrm{d}(\mathrm{u})$

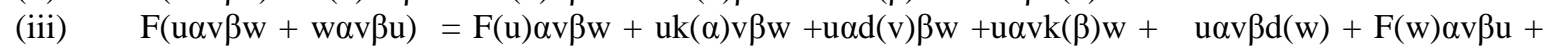
$w k(\alpha) v \beta u+\operatorname{wad}(v) \beta u+\operatorname{w\alpha vk}(\beta) u+\operatorname{wav} \beta d(u)$.

Proof: We have $u \alpha v+v \alpha u=(u+v) \alpha(u+v)-u \alpha u-v \alpha v$, and the left side as like as the right side is in U.

Hence $\quad F(u \alpha v+v \alpha u)=F((u+v) \alpha(u+v)-u \alpha u-v \alpha v)$

$=\mathrm{F}(\mathrm{u}+\mathrm{v}) \alpha(\mathrm{u}+\mathrm{v})+(\mathrm{u}+\mathrm{v}) \mathrm{k}(\alpha)(\mathrm{u}+\mathrm{v})+(\mathrm{u}+\mathrm{v}) \alpha \mathrm{d}(\mathrm{u}+\mathrm{v})-(\mathrm{F}(\mathrm{u}) \alpha \mathrm{u}+\mathrm{uk}(\alpha) \mathrm{u}+\mathrm{u} \alpha \mathrm{d}(\mathrm{u})+\mathrm{F}(\mathrm{v}) \alpha \mathrm{v}+\mathrm{vk}(\alpha) \mathrm{v}+\mathrm{v} \alpha \mathrm{d}(\mathrm{v}))$

$=F(u) \alpha u+F(u) \alpha v+F(v) \alpha u+F(v) \alpha v+u k(\alpha) u+u k(\alpha) v+v k(\alpha) u+v k(\alpha) v+u \alpha d(u)+u \alpha d(v)+v \alpha d(u)+v \alpha d(v)-$

$\mathrm{F}(\mathrm{u}) \alpha \mathrm{u}-\mathrm{uk}(\alpha) \mathrm{u}-\mathrm{u} \alpha \mathrm{d}(\mathrm{u})-\mathrm{F}(\mathrm{v}) \alpha \mathrm{v}-\mathrm{vk}(\alpha) \mathrm{v}-\mathrm{v} \alpha \mathrm{d}(\mathrm{v})$.

$\Rightarrow \mathrm{F}(\mathrm{u} \alpha \mathrm{v}+\mathrm{v} \alpha \mathrm{u})=\mathrm{F}(\mathrm{u}) \alpha \mathrm{v}+\mathrm{uk}(\alpha) \mathrm{v}+\mathrm{u} \alpha \mathrm{d}(\mathrm{v})+\mathrm{F}(\mathrm{v}) \alpha \mathrm{u}+\mathrm{vk}(\alpha) \mathrm{u}+\mathrm{v} \alpha \mathrm{d}(\mathrm{u})$.

Replacing v by $\mathrm{u} \beta \mathrm{v}+\mathrm{v} \beta \mathrm{u}$ we have,

$\mathrm{F}(\mathrm{u} \alpha(\mathrm{u} \beta \mathrm{v}+\mathrm{v} \beta \mathrm{u})+(\mathrm{u} \beta \mathrm{v}+\mathrm{v} \beta \mathrm{u}) \alpha \mathrm{u})=\mathrm{F}(\mathrm{u}) \alpha(\mathrm{u} \beta \mathrm{v}+\mathrm{v} \beta \mathrm{u})+\mathrm{uk}(\alpha)(\mathrm{u} \beta \mathrm{v}+\mathrm{v} \beta \mathrm{u})+\mathrm{u} \alpha \mathrm{d}(\mathrm{u} \beta \mathrm{v}+\mathrm{v} \beta \mathrm{u})+\mathrm{F}(\mathrm{u} \beta \mathrm{v}+\mathrm{v} \beta \mathrm{u}) \alpha \mathrm{u}+$ $(u \beta v+v \beta u) k(\alpha) u+(u \beta v+v \beta u) \alpha d(u)$.

Left side of (1) is equal to

$\mathrm{F}(\mathrm{u} \alpha \mathrm{u} \beta \mathrm{v}+\mathrm{u} \alpha \mathrm{v} \beta \mathrm{u}+\mathrm{u} \beta \mathrm{v} \alpha \mathrm{u}+\mathrm{v} \beta \mathrm{u} \alpha \mathrm{u})=\mathrm{F}(\mathrm{u} \alpha \mathrm{v} \beta \mathrm{u}+\mathrm{u} \beta \mathrm{v} \alpha \mathrm{u})+\mathrm{F}((\mathrm{u} \alpha \mathrm{u}) \beta v+\mathrm{v} \beta(\mathrm{u} \alpha \mathrm{u}))$

$=F(u \alpha v \beta u+u \beta v \alpha u)+F(u \alpha u) \beta v+u \alpha u k(\beta) v+u \alpha u \beta d(v)+F(v) \beta u \alpha u+v k(\beta) u \alpha u+\quad v \beta d(u \alpha u)$

$=\mathrm{F}(\mathrm{u} \alpha \mathrm{v} \beta \mathrm{u}+\mathrm{u} \beta \mathrm{v} \alpha \mathrm{u})+\mathrm{F}(\mathrm{u}) \alpha \mathrm{u} \beta \mathrm{v}+\mathrm{uk}(\alpha) \mathrm{u} \beta \mathrm{v}+\mathrm{u} \alpha \mathrm{d}(\mathrm{u}) \beta \mathrm{v}+\mathrm{u} \alpha \mathrm{uk}(\beta) \mathrm{v}+\mathrm{u} \alpha \mathrm{u} \beta \mathrm{d}(\mathrm{v})+\mathrm{F}(\mathrm{v}) \beta \mathrm{u} \alpha \mathrm{u}+\mathrm{vk}(\beta) \mathrm{u} \alpha \mathrm{u}+$ $v \beta d(u) \alpha u+v \beta u k(\alpha) u+v \beta u \alpha d(u)$.

Right side of (1) is equal to

$\mathrm{F}(\mathrm{u}) \alpha u \beta v+F(u) \alpha v \beta u+u k(\alpha) u \beta v+u k(\alpha) v \beta u+u \alpha d(u) \beta v+u \alpha u k(\beta) v+u \alpha u \beta d(v)+u \alpha d(v) \beta u+u \alpha v k((\beta) u+$

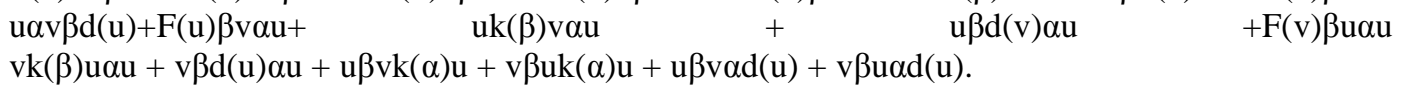


Computing both sides we have,

$\mathrm{F}(\mathrm{u} \alpha \mathrm{v} \beta \mathrm{u}+\mathrm{u} \beta \mathrm{v} \alpha \mathrm{u})=\mathrm{F}(\mathrm{u}) \alpha v \beta \mathrm{u}+\mathrm{uk}(\alpha) v \beta \mathrm{u}+\mathrm{u} \alpha \mathrm{d}(\mathrm{v}) \beta \mathrm{u}+\mathrm{u} \alpha \mathrm{vk}(\beta) \mathrm{u}+\mathrm{u} \alpha \mathrm{v} \beta \mathrm{d}(\mathrm{u})+$

$\mathrm{F}(\mathrm{u}) \beta \mathrm{v} \alpha u+\mathrm{uk}(\beta) v \alpha u+u d(v) \alpha u+u \beta v k(\alpha) u+u \beta v \alpha d(u)$.

Putting $u \beta v \alpha u=u \alpha v \beta u$ we have,

$\mathrm{F}(2 \mathrm{u} \alpha \mathrm{v} \beta \mathrm{u})=\mathrm{F}(\mathrm{u}) \alpha v \beta \mathrm{u}+\mathrm{uk}(\alpha) v \beta \mathrm{u}+\mathrm{u} \alpha \mathrm{d}(\mathrm{v}) \beta \mathrm{u}+\mathrm{u} \alpha \mathrm{vk}(\beta) \mathrm{u}+\mathrm{u} \alpha v \beta d(\mathrm{u})+\mathrm{F}(\mathrm{u}) \alpha v \beta \mathrm{u}+$

$\operatorname{u\alpha vk}(\beta) \mathrm{u}+\mathrm{u} \alpha \mathrm{d}(\mathrm{v}) \beta \mathrm{u}+\mathrm{uk}(\alpha) \mathrm{v} \beta \mathrm{u}+\mathrm{u} \alpha \mathrm{v} \beta \mathrm{d}(\mathrm{u})$

$\Rightarrow 2 \mathrm{~F}(\mathrm{u} \alpha \mathrm{v} \beta \mathrm{u})=2(\mathrm{~F}(\mathrm{u}) \alpha \mathrm{v} \beta \mathrm{u}+\mathrm{uk}(\alpha) \mathrm{v} \beta \mathrm{u}+\mathrm{u} \alpha \mathrm{d}(\mathrm{v}) \beta \mathrm{u}+\mathrm{u} \alpha \mathrm{vk}(\beta) \mathrm{u}+\mathrm{u} \alpha \mathrm{v} \beta \mathrm{d}(\mathrm{u}))$.

Since $M$ is a 2- torsion free, hence we have

$\mathrm{F}(\operatorname{u\alpha v\beta u})=\mathrm{F}(\mathrm{u}) \alpha v \beta \mathrm{u}+\mathrm{uk}(\alpha) v \beta \mathrm{u}+\mathrm{u} \alpha \mathrm{d}(\mathrm{v}) \beta \mathrm{u}+\mathrm{u} \alpha v k(\beta) \mathrm{u}+\mathrm{u} \alpha v \beta d(\mathrm{u})$.

Replace $\mathrm{u}+\mathrm{w}$ for $\mathrm{u}$ we have,

$\mathrm{F}((\mathrm{u}+\mathrm{w}) \alpha v \beta(\mathrm{u}+\mathrm{w}))=\mathrm{F}(\mathrm{u}+\mathrm{w}) \alpha v \beta(\mathrm{u}+\mathrm{w})+(\mathrm{u}+\mathrm{w}) \mathrm{k}(\alpha) v \beta(\mathrm{u}+\mathrm{w})+(\mathrm{u}+\mathrm{w}) \alpha d(\mathrm{v}) \beta(\mathrm{u}+\mathrm{w})+(\mathrm{u}+\mathrm{w}) \alpha v k(\beta)(\mathrm{u}+\mathrm{w})+$ $(\mathrm{u}+\mathrm{w}) \alpha v \beta d(\mathrm{u}+\mathrm{w})$

Left side of (2) is equal to

$F(\operatorname{u\alpha v} \beta u+\operatorname{u\alpha v} \beta w+w \alpha v \beta u+w \alpha v \beta w)=F(\operatorname{uav} \beta w+w \alpha v \beta u)+F(\operatorname{u\alpha v} \beta u)+F(\operatorname{wav} \beta w)$

$=\mathrm{F}(\operatorname{u\alpha v} \beta \mathrm{w}+\mathrm{w \alpha v} \beta \mathrm{u})+\mathrm{F}(\mathrm{u}) \alpha v \beta \mathrm{u}+\mathrm{uk}(\alpha) v \beta \mathrm{u}+\mathrm{u} \alpha \mathrm{d}(\mathrm{v}) \beta \mathrm{u}+\mathrm{u} \alpha \mathrm{vk}(\beta) \mathrm{u}+\mathrm{u} \alpha \mathrm{v} \beta \mathrm{d}(\mathrm{u})+\mathrm{F}(\mathrm{w}) \alpha v \beta \mathrm{w}+\mathrm{wk}(\alpha) v \beta \mathrm{w}+$ $\operatorname{w\alpha d}(\mathrm{v}) \beta \mathrm{w}+\operatorname{w\alpha vk}(\beta) \mathrm{w}+\operatorname{w\alpha v} \beta \mathrm{d}(\mathrm{w})$.

Right side of (2) is equal to

$\mathrm{F}(\mathrm{u}) \alpha \mathrm{v} \beta \mathrm{u}+\mathrm{F}(\mathrm{w}) \alpha \mathrm{v} \beta \mathrm{u}+\mathrm{F}(\mathrm{u}) \alpha v \beta \mathrm{w}+\mathrm{F}(\mathrm{w}) \alpha v \beta \mathrm{w}+\mathrm{uk}(\alpha) v \beta \mathrm{u}+\mathrm{wk}(\alpha) v \beta \mathrm{u}+\mathrm{uk}(\alpha) v \beta \mathrm{w}+\mathrm{wk}(\alpha) v \beta \mathrm{w}+\mathrm{u} \alpha \mathrm{d}(\mathrm{v}) \beta \mathrm{u}$

$+\operatorname{wad}(v) \beta u+\operatorname{u\alpha d}(v) \beta w+\operatorname{wad}(v) \beta w+\operatorname{u\alpha vk}(\beta) u+\operatorname{wavk}(\beta) u+\operatorname{u\alpha vk}(\beta) w+\operatorname{wavk}(\beta) w+\operatorname{u\alpha v} \beta d(u)+\operatorname{u\alpha v} \beta d(w)$

$+w \alpha v \beta d(u)+w \alpha v \beta d(w)$.

Comparing both sides we get,

$\mathrm{F}(\operatorname{u\alpha v} \beta \mathrm{w}+\operatorname{w\alpha v\beta u})=\mathrm{F}(\mathrm{u}) \alpha v \beta \mathrm{w}+\mathrm{uk}(\alpha) v \beta \mathrm{w}+\mathrm{u} \alpha \mathrm{d}(\mathrm{v}) \beta \mathrm{w}+\operatorname{u\alpha vk(\beta )w}+\mathrm{u} \alpha v \beta d(w)+F(w) \alpha v \beta u+w k(\alpha) v \beta u+$ $\operatorname{wad}(v) \beta u+\operatorname{wavk}(\beta) u+\operatorname{wav} \beta d(u)$.

Definition: We define $\psi_{\alpha}(u, v)=F(u \alpha v)-F(u) v-u k(\alpha) v-u \alpha d(v)$ for all $u, v \in U$ and $\alpha \in \Gamma$.

Remark 2.6 : It is clear that F is a generalized k- derivation if and only if $\Psi_{\alpha}(u, v)=0$.

Lemma 2.7 : Let $\mathrm{M}, \mathrm{U}$ and $\mathrm{F}$ be as in above. Then for all $\mathrm{u}, \mathrm{v}, \mathrm{w} \in \mathrm{U}$ and $\alpha, \beta \in \Gamma$, the following relations hold :

(i) $\Psi_{\alpha}(u, v)+\Psi_{\alpha}(v, u)=0$

(ii) $\Psi_{\alpha}(u+w, v)=\Psi_{\alpha}(u, v)+\Psi_{\alpha}(w, v)$

(iii) $\Psi(u, v+w)=\Psi_{\alpha}(u, v)+\Psi_{\alpha}(u, w)$

(iv) $\Psi_{\alpha+\beta}(\mathrm{u}, \mathrm{v})=\Psi_{\alpha}(\mathrm{u}, \mathrm{v})+\Psi_{\beta}(\mathrm{u}, \mathrm{v})$.

Lemma 2.8 : Let $\mathrm{M}, \mathrm{U}, \mathrm{F}$ and $\mathrm{d}$ be defined as in above, then for all $\mathrm{u}, \mathrm{v}, \mathrm{w} \in \mathrm{U}$ and $\alpha, \beta, \gamma \in \Gamma$, $\Psi_{\alpha}(u, v) \beta w \gamma[u, v]_{\alpha}=0$.

Proof: Consider $A=(2 u \alpha v) \beta w \gamma(2 v \alpha u)+(2 v \alpha u) \beta w \gamma(2 u \alpha v)$.

From Lemma 2.4 (iii) we have, $\quad F(A)=F((2 u \alpha v) \beta w \gamma(2 v \alpha u)+(2 v \alpha u) \beta w \gamma(2 u \alpha v))$

$=F(2 u \alpha v) \beta w \gamma(2 v \alpha u)+2 u \alpha v k(\beta) w \gamma(2 v \alpha u)+2 u \alpha v \beta d(w) \gamma(2 v \alpha u)+2 u \alpha v \beta w k(\gamma)(2 v \alpha u)+$ $(2 u \alpha v) \beta w \gamma d(2 v \beta u)+F(2 v \alpha u) \beta w \gamma(2 u \alpha v)+(2 v \alpha u) k(\beta) w \gamma(2 u \alpha v)+(2 v \alpha u) \beta d(w) \gamma(2 u \alpha v)+$ $(2 v \alpha u) \beta w k(\gamma)(2 u \alpha v)+(2 v \alpha u) \beta w \gamma d(2 u \alpha v)$

$=4[F(u \alpha v) \beta w \gamma(v \alpha u)+u \alpha v k(\beta) w \gamma \alpha u+u \alpha v \beta d(w) v \alpha u+u \alpha v \beta w k(\gamma) v \alpha u+u \alpha v \beta w \gamma d(v \beta u)+$ $F(v \alpha u) \beta w \gamma u \alpha v+v \alpha u k(\beta) w \gamma u \alpha v+v \alpha u \beta d(w) \mu u \alpha v+v \alpha u \beta w k(\gamma) u \alpha v+v \beta u \beta w \gamma d(u \alpha v)]$.

Again $A=(2 u \alpha v) \beta w \gamma(2 v \alpha u)+(2 v \alpha u) \beta w \gamma(2 u \alpha v)=u \alpha(4 v \beta w \gamma) \alpha u+v \alpha(4 u \beta w \gamma) \alpha v$ 
$\Rightarrow F(A)=F(u \alpha(4 v \beta w \gamma) \alpha u+v \alpha(4 u \beta w \gamma u) \alpha v)$

$=4[F(u) \alpha v \beta w w \alpha u+u k(\alpha) v \beta w v \alpha u+u \alpha d(v \beta w \gamma) \alpha u+u \alpha v \beta w \gamma k(\alpha) u+u \alpha v \beta w v \alpha d(u)+$

$F(v) \alpha u \beta w \gamma u \alpha v+v k(\alpha) u \beta w \gamma u \alpha v+v \alpha d(u \beta w \gamma u) \alpha v+v \alpha u \beta w \gamma u k(\alpha) v+v \alpha u \beta w \gamma u \alpha d(v)]$ using lemma 2.4(ii)

$=4[F(u) \alpha v \beta w \gamma \alpha u+u k(\alpha) v \beta w v v \alpha u+u \alpha d(v) \beta w v \alpha u+u \alpha v k(\beta) w \gamma \alpha u+u \alpha v \beta d(w) w \alpha u+$

$u \alpha v \beta w k(\gamma) v \alpha u+u \alpha v \beta w \gamma d(v) \alpha u+u \alpha v \beta w \gamma k(\alpha) u+u \alpha v \beta w v \alpha d(u)+F(v) \alpha u \beta w \gamma u \alpha v+$

$v k(\alpha) u \beta w \eta u \alpha v+v \alpha d(u) \beta w \gamma \alpha v+v \alpha u k(\beta) w \gamma u \alpha v+v \alpha u \beta d(w) \mu \alpha v+v \alpha u \beta w k(\gamma) u \alpha v+$

$v \alpha u \beta w \gamma d(u) \alpha v+v \alpha u \beta w \gamma u k(\alpha) v+v \alpha u \beta w \mu \alpha d(v)]$

Comparing both expressions we have,

$4[F(u \alpha v) \beta w v \alpha u+F(v \alpha u) \beta w \gamma u \alpha v+u \alpha v \beta w \gamma d(v \alpha u)+v \alpha u \beta w \gamma d(u \alpha v)]=4[F(u) \alpha v \beta w \gamma \alpha u+$ $u k(\alpha) v \beta w v \alpha u+u \alpha d(v) \beta w \gamma \alpha u+u \alpha v \beta w \gamma d(v) \alpha u+u \alpha v \beta w \gamma k(\alpha) u+u \alpha v \beta w \gamma \alpha d(u)+F(v) \alpha u \beta w \gamma u \alpha v+$ $v k(\alpha) u \beta w \gamma u \alpha v+v \alpha d(u) \beta w \eta u \alpha v+v \alpha u \beta w \gamma d(u) \alpha v+v \alpha u \beta w \gamma u k(\alpha) v+v \alpha u \beta w \gamma u \alpha d(v)]$

Since $M$ is a 2- torsion free, we have

$[F(u \alpha v)-F(u) \alpha v-u k(\alpha) v-u \alpha d(v)] \beta w \gamma \alpha u+[F(v \alpha u)-F(v) \alpha u-v k(\alpha) u-v \alpha d(u)] \beta w \gamma u \alpha v+$ $u \alpha v \beta w \gamma[d(v \alpha u)-d(v) \alpha u-v k(\alpha) u-v \alpha d(u)]+v \alpha u \beta w \gamma[d(u \alpha v)-d(u) \alpha v-u k(\alpha) v-u \alpha d(v)]=0$

$\Rightarrow \psi_{\alpha}(u, v) \beta w \gamma \alpha u+\psi_{\alpha}(v, u) \beta w \gamma u \alpha v+[d(v \alpha u)-d(v \alpha u)]+[d(u \alpha v)-d(u \alpha v)]=0$

$\Rightarrow \psi_{\alpha}(u, v) \beta w \gamma \alpha u-\psi_{\alpha}(u, v) \beta w \gamma u \alpha v=0$

$\Rightarrow-\psi_{\alpha}(u, v) \beta w \gamma(v \alpha u-u \alpha v)=0$

$\Rightarrow \psi_{\alpha}(u, v) \beta w \gamma[u, v]_{\alpha}=0$

Lemma 2.9: [16, Lemma 2.10] Let $U \not \subset Z(M)$ be a Lie ideal of a Prime $\Gamma$ - ring $M$ satisfying the condition (*) and $\mathrm{a}, \mathrm{b} \in \mathrm{M}($ res. $\mathrm{b} \in \mathrm{U}$ and $\mathrm{a} \in \mathrm{M})$ such that $\mathrm{a} \alpha \mathrm{U} \beta \mathrm{b}=0$ for all $\alpha, \beta \in \Gamma$, then $\mathrm{a}=0$, or $\mathrm{b}=0$.

Lemma 2.10 : Let $\mathrm{U} \not \subset \mathrm{Z}(\mathrm{M})$ be a Lie ideal of a 2 - torsion free prime $\Gamma$ - ring $\mathrm{M}$. Then $[u, v]_{\alpha} \beta w \gamma \psi_{\alpha}(u, v)=0$.

Proof: From Lemma 2.8 we have, $\psi_{\alpha}(u, v) \delta x \mu[u, v]_{\alpha}=0$

$\Rightarrow[u, v]_{\alpha} \beta w \gamma \psi_{\alpha}(u, v) \delta x \mu[u, v]_{\alpha} \beta w \gamma \psi_{\alpha}(u, v)=0$ for all $x \in U$.

In view of Lemma 2.9, we have $[u, v]_{\alpha} \beta w \gamma \psi_{\alpha}(u, v)=0$.

Lemma 2.11: Let $\mathrm{U} \not \subset \mathrm{Z}(\mathrm{M})$ be a Lie ideal of a 2- torsion free prime $\Gamma$-ring . Then $\psi_{\alpha}(u, v) \beta w \gamma[x, y]_{\delta}=0$ for all $\mathrm{u}, \mathrm{v}, \mathrm{w}, \mathrm{x}, \mathrm{y} \in \mathrm{U}$ and $\alpha, \beta, \gamma, \delta \in \Gamma$.

Proof: From Lemma 2.8 we have $\psi_{\alpha}(u+x, v) \beta w \gamma[u+x, v]_{\alpha}=0$

$\Rightarrow \psi_{\alpha}(u, v) \beta w \gamma[u, v]_{\alpha}+\psi_{\alpha}(u, v) \beta w \gamma[x, v]_{\alpha}+\psi_{\alpha}(x, v) \beta w \gamma[u, v]_{\alpha}+\psi_{\alpha}(x, v) \beta w \gamma[x, v]_{\alpha}=0$

$\Rightarrow \Psi_{\alpha}(u, v) \beta w \gamma[x, v]_{\alpha}+\Psi_{\alpha}(x, v) \beta w \gamma[u, v]_{\alpha}=0$

$\Rightarrow \psi_{\alpha}(u, v) \beta w \gamma[x, v]_{\alpha}=-\psi_{\alpha}(x, v) \beta w \gamma[u, v]_{\alpha}$

$\Rightarrow\left(\psi_{\alpha}(u, v) \beta w \gamma[x, v]_{\alpha}\right) \partial p \theta\left(\psi_{\alpha}(u, v) \beta w \gamma[x, v]_{\alpha}\right)$

$=-\psi_{\alpha}(x, v) \beta w \gamma[u, v]_{\alpha} \delta p \theta \psi_{\alpha}(u, v) \beta w \gamma[x, v]_{\alpha}$

$=-\psi_{\alpha}(x, v) \beta w \gamma\left([u, v]_{\alpha} \delta p \theta \psi_{\alpha}(u, v)\right) \beta w \gamma[x, v]_{\alpha}=0 \quad$ [by lemma 2.8] 
By Lemma 2.9, we have $\psi_{\alpha}(u, v) \beta w \gamma[x, v]_{\alpha}=0$

Likewise by replacing $\mathrm{v}+\mathrm{y}$ for $\mathrm{v}$ we get $\psi_{\alpha}(u, v) \beta w \gamma[x, y]_{\alpha}=0$.

Proceeding in the same way as above, by the similar replacement in the result, we have $[x, y]_{\alpha} \beta w \gamma \psi_{\alpha}(u, v)=0$

Now putting $\alpha+\delta$ for $\alpha$ in (i) we have, $\psi_{\alpha+\delta}(u, v) \beta w \gamma[x, y]_{\alpha+\delta}=0$

$\Rightarrow \psi_{\alpha}(u, v) \beta w \gamma[x, y]_{\alpha}+\psi_{\delta}(u, v) \beta w \gamma[x, y]_{\alpha}+\psi_{\alpha}(u, v) \beta w \gamma[x, y]_{\delta}+\psi_{\delta}(u, v) \beta w \gamma[x, y]_{\delta}=0$

$\Rightarrow \psi_{\delta}(u, v) \beta w \gamma[x, y]_{\alpha}+\psi_{\alpha}(u, v) \beta w \gamma[x, y]_{\delta}=0$

$\Rightarrow \psi_{\alpha}(u, v) \beta w \gamma[x, y]_{\delta}=\psi_{\delta}(u, v) \beta w \gamma[x, y]_{\alpha}$

Therfore,

$\psi_{\alpha}(u, v) \beta w \gamma[x, y]_{\delta} \theta q \mu \psi_{\alpha}(u, v) \beta w \gamma[x, y]_{\delta}=-\psi_{\delta}(u, v) \beta w \gamma\left([x, y]_{\alpha} \theta q \mu \psi_{\alpha}(u, v)\right) \beta w \gamma[x, y]_{\delta}=0$ by (i)

Using Lemma 2.9, we have $\psi_{\alpha}(u, v) \beta w \gamma[x, y]_{\delta}=0$.

Lemma 2.12: Let $U \not \subset Z(M)$ be a Lie ideal of a 2- torsion free prime $\Gamma$ - ring $M$, then $Z(U)=Z(M)$.

Proof: We have $Z(U)$ is both a sub $\Gamma$-ring and a Lie ideal of $M$. Also we know that $Z(U)$ cannot contain a nonzero ideal of $M$. So by [17, Lemma 3.7], $Z(U)$ is contained in $Z(M)$. Therefore, $Z(U)=Z(M)$.

Lemma 2.13: Let $\mathrm{U} \not \subset \mathrm{Z}(\mathrm{M})$ be a Lie ideal of a 2- torsion free prime $\Gamma$ - ring $\mathrm{M}$. Then $\psi_{\alpha}(u, v) \in Z(U)=Z(M)$ for every $u, v \in U, \alpha \in \Gamma$.

Proof : We have $\psi_{\alpha}(u, v) \beta w \gamma\left([x, y]_{\delta}\right)=0$

Now $2\left[\psi_{\alpha}(u, v), c\right]_{\delta} \beta w \gamma\left[\psi_{\alpha}(u, v), c\right]_{\delta}$

$\left.=2 \psi_{\alpha}(u, v) \delta c-c \delta \psi_{\alpha}(u, v)\right) \beta w \gamma\left[\psi_{\alpha}(u, v), c\right]_{\delta}$

$=\psi_{\alpha}(\mathrm{u}, \mathrm{v}) \delta(2 c \beta w) \gamma\left[\psi_{\alpha}(u, v), c\right]_{\delta}-2 c \delta \psi_{\alpha}(u, v) \beta w \gamma\left[\psi_{\alpha}(u, v), c\right]_{\delta}=0$, for every $c \in U$.

In view of Lemma 2.9, we have $\left[\Psi_{\alpha}(u, v), c\right]_{\delta}=0$,

$\Rightarrow \psi_{\alpha}(u, v) \in Z(U)$ and that implies $\psi_{\alpha}(u, v) \in Z(M)$ by Lemma 2.12.

Lemma 2.14: Let $M$ be a 2- torsion free prime $\Gamma$ - ring satisfying the condition $\left(^{*}\right)$ and $U$ a Lie ideal of $M$. Let $u \in U$ be such that $\left[u .[u, x]_{\alpha}\right]_{\alpha}=0$ for all $x \in M$ and $\alpha \in \Gamma$. Then $[u, x]_{\alpha}=0$

Proof: We have $\left[u,[u, x]_{\alpha}\right]_{\alpha}=0$ for all $x \in M$ and $\alpha \in \Gamma$.

Let $\mathrm{y} \in \mathrm{M}$, then $\mathrm{x} \alpha \mathrm{y} \in \mathrm{M}$ for all $\alpha \in \Gamma$. Replace $\mathrm{x}$ by x $\alpha \mathrm{y}$ we have $\left[u,[u, x \alpha y]_{\alpha}\right]_{\alpha}=0$

$\Rightarrow\left[u,\left(x \alpha[u, y]_{\alpha}+[u, x]_{\alpha}\right) \alpha y\right]_{\alpha}=0$

$\Rightarrow\left[u, x \alpha[u, y]_{\alpha}\right]_{\alpha}+\left[u,[u, x]_{\alpha} \alpha y\right]_{\alpha}=0$

$\Rightarrow x \alpha\left[u,[u, y]_{\alpha}\right]_{\alpha}+[u, x]_{\alpha} \alpha[u, y]_{\alpha}+[u, x]_{\alpha} \alpha[u, y]_{\alpha}+\left[u,[u, x]_{\alpha}\right]_{\alpha} \alpha y=0$

$\Rightarrow 2[u, x]_{\alpha} \alpha[u, y]_{\alpha}=0$

Since $\mathrm{M}$ is 2 - torsion free, we have $[u, x]_{\alpha} \alpha[u, y]_{\alpha}=0$.

Putting $\mathrm{y}=\mathrm{u} \beta \mathrm{x}$, we have $[u, x]_{\alpha} \alpha[u, u \beta x]_{\alpha}=0 \Rightarrow[u, x]_{\alpha} \alpha u \beta[u, x]_{\alpha}=0$ by using $\left(^{*}\right)$.

Hence by Lemma 2.9 we have $[u, x]_{\alpha}=0$.

Lemma 2.15 : Let $M$ be a 2-torsion free prime $\Gamma$ - ring satisfying the condition $\left(^{*}\right)$ and $U$ be a commutative Lie ideal of $\mathrm{M}$, then $\mathrm{U} \subseteq \mathrm{Z}(\mathrm{M})$. 
Proof : Since U is commutative, we have $[u, v]_{\alpha}=0$ for all $u, v \in U$ and $\alpha \in \Gamma$.

Also we have $[u, x]_{\alpha} \in U$ for all $u \in U, x \in M$ and $\alpha \in \Gamma$.

Replacing $v$ by $[u, x]_{\alpha}$, we obtain $\left[u,[u, x]_{\alpha}\right]_{\alpha}=0$

By Lemma 2.14 we have $[u, x]_{\alpha}=0$. Hence $U \subseteq Z(M)$.

Theorem 2.16: Let $M$ be a 2- torsion free prime $\Gamma$-ring satisfying the condition $\left(^{*}\right)$ and let $\mathrm{U}$ be a Lie ideal of $\mathrm{M}$ such that $\mathrm{u} \alpha \mathrm{u} \in \mathrm{U}$ for all $\mathrm{u} \in \mathrm{U}$ and if $\mathrm{F}: \mathrm{M} \rightarrow \mathrm{M}$ is a Jordan generalized derivation on $\mathrm{U}$ of $\mathrm{M}$ then,

$\psi_{\alpha}(u, v)=0$ for all $\mathrm{u}, \mathrm{v} \in \mathrm{U}$ and $\alpha \in \Gamma$.

Proof: Let $\mathrm{U}$ be a commutative Lie ideal of $\mathrm{M}$. Then by Lemma 2.14, $U \subseteq Z(M)$.

Since $\mathrm{U}$ is commutative, then $[v, w]_{\beta}=0$ implies $v \alpha w=w \alpha v$, for every $v, w \in U, \alpha \in \Gamma$.

From Lemma 2.4 (iii) we have,

$F(u \alpha v \beta w+w \alpha v \beta u)=F(u) \alpha v \beta w+u k(\alpha) v \beta w+u \alpha d(v) \beta w+u \alpha v k(\beta) w+u \alpha v \beta d(w)+F(w) \alpha v \beta u+$

$w k(\alpha) v \beta u+w \alpha d(v) \beta u+w \alpha v k(\beta) u+w \alpha v \beta d(u)$

Putting $\mathrm{u}=2 \mathrm{v} \beta \mathrm{w}$ in (1), we have

L.S. $=F(2 v \beta w \alpha v \beta w+w \alpha v \beta 2 v \beta w)$

$=2 F(v \beta w \alpha v \beta w+w \beta v \alpha v \beta w)$

$=2 F(v \beta w \alpha v \beta w+v \beta w \alpha v \beta w)$

$=4 F((v \beta w) \alpha(v \beta w))$

$=4(F(v \beta w) \alpha(v \beta w)+v \beta w k(\alpha) v \beta w+v \beta w \alpha d(v \beta w))$.

Also R.S. $=F(2 v \beta \beta) \alpha v \beta w+2 v \beta \beta w(\alpha) v \beta w+2 v \beta w \alpha d(v) \beta w+2 v \beta w \alpha v k(\beta) w+2 v \beta w \alpha v d(w)+$ $F(w) \alpha v \beta 2 v \beta w+w k(\alpha) v \beta 2 v \beta w+w \alpha d(v) \beta 2 v \beta w+w \alpha v k(\beta) 2 v \beta w+w \alpha v \beta d(2 v \beta w)$

$=F(2 v \beta w) \alpha v \beta w+2 v \beta w k(\alpha) v \beta w+2 v \beta w \alpha[d(v) \beta w+v k(\beta) w+v \beta d(w)]+2 F(w) \alpha v \beta v \beta w+$

$2 w k(\alpha) v \beta v \beta w+2 w \alpha d(v) \beta v \beta w+2 w \alpha v k(\beta) v \beta w+2 w \alpha v \beta d(v \beta w)$

$=2 F(v \beta w) \alpha v \beta w+2 v \beta w k(\alpha) v \beta w+4 v \beta w \alpha d(v \beta w)+2 F(w) \beta v \alpha v \beta w+2 w k(\alpha) v \beta v \beta w+2 w \alpha d(v) \beta v \beta w+$ $w k(\beta) v \alpha v \beta w$.

Comparing both sides we get

$2 F(v \beta w) \alpha v \beta w+2 v \beta w k(\alpha) v \beta w-2 F(w) \beta v \alpha v \beta w-2 w k(\beta) v \alpha v \beta w-2 w \beta d(v) \alpha v \beta w-2 v \beta w k(\alpha) v \beta w=0$

$\Rightarrow 2(F(w \beta v)-F(w) \beta v-w k(\beta) v-w \beta d(v)) \alpha v \beta w=0$

$\Rightarrow 2 \psi_{\beta}(w . v) \alpha v \beta w=0 \Rightarrow \psi_{\beta}(w, v) \alpha v \beta w=0$

$\Rightarrow \psi_{\beta}(w, v) \alpha v \beta w \gamma x \delta y=0$, where $x \in U, y \in M$.

$\Rightarrow \psi_{\beta}(w, v) \alpha x \beta y \gamma \delta w=0$

$\Rightarrow\left(\psi_{\beta}(w, v) \alpha x \gamma y\right) \beta v \delta w=0 \quad \operatorname{using}(*)$.

From Lemma 2.9, either $\psi_{\beta}(w, v) \alpha x \gamma y=0$ or $w=0$.

Since $w \in U, w \neq 0$, hence $\psi_{\beta}(w, v) \alpha x \gamma y=0$. That implies $\psi_{\beta}(w, v) \alpha U \gamma y=0$.

Using Lemma 2.9 we have, $\psi_{\beta}(w, v)=0$.

Again if $\mathrm{U}$ is not commutative, i.e., $U \not \subset Z(M)$, then from Lemma 2.11 we have

$\psi_{\alpha}(u, v) \beta w \gamma[x, y]_{\delta}=0$. But $[x, y]_{\delta}=0$ implies $U \subseteq Z(M)$, a contradiction .

Hence $\psi_{\alpha}(u, v)=0$, for all $u, v \in U$ and $\alpha \in \Gamma$. 
Corollary 2.17: Every Jordan generalized k-derivation of a 2 -torsion free prime $\Gamma$ - ring $M$ is a generalized kderivation on $\mathrm{M}$.

\section{References}

[1] N. Nobusawa, On the generalization of ring theory, Osaka J . Math 1 (1964), 81 - 89.

[2] W. E. Barnes, On the $\Gamma$ - rings of Nobusawa, Pacific J. Math. , 18(1966) , 411 - 422.

[3] M. Sapanci and A. Nakajima, Jordan derivations on completely Prime $\Gamma$ - rings , Math Japanica 46 (1997), 47 - 51

[4] H. Kandamar , The k- derivations of a gamma ring, Turkish J. Math. , 24 (2000), 221 - 231.

[5] S. Chakraborty and A. C. Paul, On Jordan k-derivations of a 2-torsion free Prime $\Gamma N$ - rings, Punjab Univ. J. Math . vol. 40(2008), 97 - 101.

[6] Y. Ceven and M. A . Ozturk, on Jordan generalized derivations in Gamma rings , Itacezzepe J. Math . and staf , 33 (2004) , 11 14

[7] M. M. Rahman and A. C. Paul, "Jodan generalized derivations on Lie ideals of prime $\Gamma$ - rings". South Asian Journal of Mathematics, 2013, vol 3(3): $148-153$.

[8] A. C. Paul and Md . Sabur Uddin, Simple Gamma rings with involutions, IOSR Journal of Mathematics vol. 4, Issue 3 (Nov Dec 2012 ) PP $40-48$.

[9] I. N. Herstein, Topics in Ring Theory, The University of Chicago Press, Chicago, 111. London , 1969.

[10] S. Chakraborty and A. C . Paul, k-Derivations and k- homomorphisms of Gamma rings , Lambert Academic Publishing CmbH Co, KG Heinrich - Beking - Str . 6- 8, 66121, saarbrchen, Germany, 2012.

[11] S. Chakraborty and A. C . Paul, on Jordan generalized k- derivations of semiprime $\Gamma$ N - rings , Bull. Iranian Math . Soe. vol. 36, No. 1 (2010), $41-53$.

[12] S. Chakraborty and A. C . Paul, Jordan generalized k- derivations of completely semiprime $\Gamma \mathrm{N}$ - rings , Bull . Allahabad Math. Soc. , V . 24, Part 1, 2009, $21-30$.

[13] S. Chakraborty and A. C . Paul, On Jordan generalized k-derivations of 2 - torsion free prime $\Gamma \mathrm{N}$ - rings , International Mathematical Forum, vol. 2 , No. 57 (2007), 2823 - 2829.

[14] S. Chakraborty and A. C . Paul, Jordan k- derivations of completely prime $\Gamma \mathrm{N}$ - rings ,Southeast Asian Bulletin of Math. , vol . $35,(2011), 29-34$

[15] S. Chakraborty and A. C . Paul, Jordan k- derivations of certain Nobusawa $\Gamma$ - rings, GANIT: Journal of Bangladesh Mathematical Soc., vol . 31, 920110, $53-64$.

[16] M. M. Rahman and A. C. Paul, Jordan Derivations on Lie ideals of Prime - rings. Mathematical Theory and Modeling (2013), vol. 3(3); $128-135$.

[17] A. C. Paul and Sabur Uddin, Lie and Jordan structure in simple gamma rings, J. Physical Sciences, 14(2010), 77 - 86. 